\title{
COHOMOLOGY OF LIE GROUPS MADE DISCRETE
}

\author{
Pere Pascual Gainza*
}

Abstract

We give a survey of the work of Milnor, Friedlander, Mislin, Suslin, and other authors on the Friedlander-Milnor conjecture on the homology of Lie groups made discrete and its relation to the algebraic $K$-theory of fields.

Let $G$ be a Lie group and let $G^{\delta}$ denote the same group with the discrete topology. The natural homomorphism $G^{\delta} \longrightarrow G$ induces a continuous map between classifying spaces

$$
\eta: B G^{\delta} \longrightarrow B G
$$

E.Friedlander and J. Milnor have conjectured that $\eta$ induces isomorphisms of homology and cohomology with finite coefficients. The homology of $B G^{\delta}$ is the Eilenberg-McLane homology of the group $G^{6}$, hard to compute, and one of the interests of the conjecture is that it permits the computation of these groups with finite coefficients through the computation (much better understood) of the homology and cohomology of $B G$.

The Eilenberg-McLane homology groups of a topological group $G$ are of interest in a variety of contexts such as the theory of foliations [3], [11], the scissors congruence [6] and algebraic $K$-theory [26]. For example, the Haefliger classifying space of the theory of foliations is closely related to the group of homeomorphisms of a topological manifold, for which one can prove analogous results of the Friedlander-Milnor conjecture with entire coefficients, of. [20], [32].

These notes are an exposition of the context of the conjecture, some known results mainly due to Milnor, Friedlander, Mislin and Suslin, and its application to the study of the groups $K_{i}(\mathrm{C}), \quad i \geq 0$.

We thank F. Guillén, V. Navarro Aznar and A. Roig for many helpful conversations about this theme. 


\section{Classifying spaces}

(1.1) Let $G$ be a topological group. Remember that a principal $G$-bundle consists of a continuous map

$$
p: E \longrightarrow B
$$

with fiber $G$, and a right $G$-action

$$
E \times G \longrightarrow E
$$

such that there is an open covering $\left\{U_{\alpha}\right\}$ of $B$ and homeomorphisms

$$
\varphi_{\alpha}: U_{\alpha} \times G \longrightarrow p^{-1}\left(U_{\alpha}\right)
$$

satisfying

$$
\begin{aligned}
p \varphi_{\alpha} & =\left.p\right|_{U_{\alpha}} \\
\varphi_{\alpha}(b, g) & =\varphi_{\alpha}(b, e) g .
\end{aligned}
$$

The notion of equivalence of principal $G$-bundles is the obvious one.

Deftnition. A classifying space for $G$ is a topological space $B G$ with a principal $G$-bundle

$$
E G \longrightarrow B G
$$

such that $E G$ is contractible and is universal in the following sense: if $p$ : $E \longrightarrow B$ is any principal $G$-bundle then there is a continuous map $B \longrightarrow B G$ such that $p$ is the fiber product

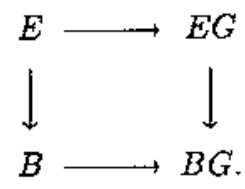

The existence of classifying spaces may be proved by Brown's representation theorem (for $C W$-complexes [38] (11.33)) or by giving a specific construction. In the following paragraphs we present Segal's construction, [30], for which we have to assume that $G$ is an ANR, as veriffed by lie groups. This construction corresponds to the nonhomogeneous normalized bar construction for discrete groups. The analogous of nonnormalized bar constructions would be the Milnor classifying space (c.f. [15]) and that of Dold-Lashof (c.f. [4]), see [33].

(1.2) Let $\mathcal{C}$ be a topological category (i.e. $O b \mathcal{C}$ and $M$ or $\mathcal{C}$ are topological spaces and the structural maps are continuous), Segal defines the simplicial topological space $N \mathcal{C}$, called the nerve of $\mathcal{C}$, whose $n$-simplexes are the elements 
$\left(f_{1}, \ldots, f_{n}\right)$ of $(\text { Mor } \mathcal{C})^{\times n}$ for which it is defined the compositions $f_{i+1} \circ f_{i}$, with boundary and degeneracy maps as usual.

Definition. $B C:=\|N \mathcal{C}\|$.

(Observe that we take the thick geometric realisation identifying only boundary maps [31]) .

To a topological group $G$ we can associate the categories:

- $G$ : it has only one object, $e \in G$, and $M$ or $G=G$,

- $\bar{G}: O b \bar{G}=G$ and $M o r \bar{G}=G \times G$,

so we obtain the classifying spaces $B G$ and $B \bar{G}$. Observe that if $g_{1}, g_{2}$ are objects of $\bar{G}$ there is one and only one morphism from $g_{1}$ to $g_{2}$ that is an isomorphism, so it follows that $\bar{G}$ is equivalent to the trivial category with one object and one morphism and from the general theory of classifying spaces we can deduce that $B \bar{G}$ is contractible. The functor $\bar{G} \longrightarrow G$ sending the morphism $\left(g_{1}, g_{2}\right)$ of $\bar{G}$ to the morphism $g_{1}^{-1} g_{2}$ of $G$, gives rise to a continuous map

$$
B \bar{G} \longrightarrow B G \text {. }
$$

Observe also that $B \bar{G}$ is a $G$-free space and that $B \bar{G} / G=B G$. We write $E G=B \bar{G}$.

Proposition. If $(G, e)$ is an $A N R$, then $E G \rightarrow B G$ is a principal $G$-bundle.

(1.3) We will give now an approximation to the universality of this principal $G$-bundle. Remember that to a principal $G$-bundle $p: E \longrightarrow B$ there are associated transition functions

$$
g_{\alpha \beta}: U_{\alpha} \cap U_{\beta} \longrightarrow G,
$$

satisfying the usual cocicle condition defined in the following way:

The maps

$$
\psi_{\alpha \beta}=\varphi_{\alpha}^{-1} \circ \varphi_{\beta}:\left(U_{\alpha} \cap U_{\beta}\right) \times G \longrightarrow\left(U_{\alpha} \cap U_{\beta}\right) \times G
$$

are compatible with the projection $p$, so they define maps

$$
h_{\alpha \beta}:\left(U_{\alpha} \cap U_{\beta}\right) \times G \longrightarrow G
$$

and as the $\varphi_{\alpha}$ are $G$-equivariant, we have

$$
\begin{aligned}
\varphi_{\alpha}\left(b, h_{\alpha \beta}(b, g)\right) & =\varphi_{\beta}(b, g) \\
& =\varphi_{\beta}(b, e) g \\
& =\varphi_{\alpha}\left(b, h_{\alpha \beta}(b, e)\right) g \\
& =\varphi_{\alpha}\left(b, h_{\alpha \beta}(b, e) g\right)
\end{aligned}
$$


i.e.

$$
h_{\alpha \beta}(b, g)=h_{\alpha \beta}(b, e) g
$$

so we can define

$$
g_{\alpha \beta}(b)=h_{\alpha \beta}(b, e) \in G .
$$

It is well known that the equivalence classes of principal $G$-bundles are determined by the transition functions (cf. by example [38] (11.16)). The information given by these functions may be interpreted in the context of classifying spaces in the following way:

Let $p: E \longrightarrow X$ be a principal $G$-bundle and let $\mathcal{U}=\left(U_{\alpha}\right)$ be an open cover of $X$ with associated transition functions $g_{\alpha \beta}$. To the couple $(X, U)$ we associate the following topological category $X_{U}$ : the objects are the pairs $\left(x, U_{\alpha}\right)$, with $x \in U_{\alpha}$, and there is a unique morphism $\left(x, U_{\alpha}\right) \longrightarrow\left(y, U_{\beta}\right)$ iff $x=y$, i.e. ,

$$
\begin{aligned}
\text { ob } X_{U} & =\coprod_{\alpha} U_{\alpha} \\
\text { Mor } X_{U} & =\coprod_{\langle\alpha, \beta)} U_{\alpha} \cap U_{\beta}
\end{aligned}
$$

Observe that

$$
\left(N X_{U}\right)_{n}=\coprod_{\left(\alpha_{0} \cdots \alpha_{n}\right)} U_{\alpha_{0}} \cap \cdots \cap U_{\alpha_{n}}
$$

the sum being over all $(n+1)$-uples with

$$
U_{\alpha_{0}} \cap \cdots \cap U_{\alpha_{n}} \neq 0
$$

Lenma. The transition functions define a continuous functor

$$
g: X_{U} \longrightarrow G
$$

hence there is a continuous map

$$
B g: B X_{U} \longrightarrow B G
$$

Similarly, if $\mathcal{V}=\left\{V_{\alpha}=p^{-1}\left(U_{\alpha}\right)\right\}$, the trivializations $\left(\varphi_{\alpha}\right)$ define a functor

$$
E_{V} \longrightarrow \bar{G}
$$

and we obtain a continuous map

$$
B E_{V} \longrightarrow E G
$$


Finally, the projection $p$ gives rise to a functor $E_{V} \longrightarrow X_{U}$ so that the following diagram commutes

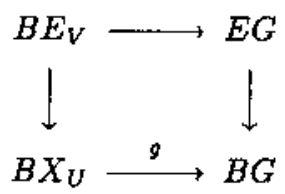

The inclusion $U \rightarrow X$ defines a morphism between simplicial spaces $N X_{U} \rightarrow$ $X$, and we obtain the commutative diagram

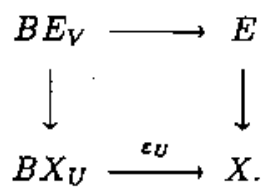

Proposition. (cf. $[30,4.1])$ If $\mathcal{U}$ is a numerable covering, then the natural map

$$
B X_{U} \longrightarrow X
$$

is a homotopy equivalence.

For a paracompact space each covering is numerable so we deduce

Corollary. $E G \longrightarrow B G$ is a classifying space of $G$ for paracompact spaces.

If $X$ is not paracompact we still have a relation between $X$ and $X_{U}$ :

Proposition. (cf. [5, p. 85]) The induced morphism

$$
H^{*}(X, Z) \longrightarrow H^{*}\left(B X_{U}, Z\right)
$$

is an isomorphism.

(1.4) If we denote by $K_{G}(X)$ the set of equivalence classes of principal $G$-bundles with base $X$, remember that a characteristic class is a natural transformation of functors

$$
c: K_{G}(-) \longrightarrow H^{*}(-, Z)
$$

The results above give: 
Proposition. The map that sends a characteristic class $c$ to the element $c(E G)$ of $H^{*}(B G, Z)$ is a bijective correspondence.

In fact, if $c$ is a characteristic class and $E \longrightarrow X$ is a principal $G$-bundle we have (notations as (1.3))

$$
\varepsilon_{U}^{*}(c(E))=g^{*}(c(E G)),
$$

but $\varepsilon_{U}^{*}$ is an isomorphism, so $c(E)$ and this identity determine $c(E G)$ completely.

Reciprocally, if $c_{0} \in H^{*}(B G, Z)$ and $E \longrightarrow X$ is a principal $G$-bundle, we can define the characteristic class $c(E)$ by

$$
\varepsilon_{U}^{*}(c(E))=g^{*}\left(c_{\theta}\right)
$$

The proof that the class so defined is independent of the trivializations and the covering $U$ may be seen in [5], pp.86-88.

(1.5) Remark that for some groups $G$ it is possible to compute the cohomology of $B G$ by Borel's theorem on the spectral sequence of the fibration $E G \longrightarrow B G$ (cf. [38], 15.62).

\section{The Weil homomorphism: The case of rational coefficients}

In this and the following $\S$ we denote by $G$ a Lie group. In this paragraph we show that the isomorphsim conjecture has not sense if we consider rational coefficients.

(2.1) Let $M$ be a differentiable manifold, $p: E \longrightarrow M$ a differentiable principal $G$-bundle and $x \in E$. The map

$$
\begin{aligned}
& G \longrightarrow E \\
& g \longmapsto x g
\end{aligned}
$$

induces an injection

$$
\nu_{x}: \mathrm{g}=T_{e} G \longrightarrow T_{x} E,
$$

such that the sequence

$$
0 \longrightarrow \mathrm{g} \stackrel{\nu_{x}}{\longrightarrow} T_{x} E \stackrel{d p_{x}}{\longrightarrow} T_{p(x)} M \longrightarrow 0
$$

is exact.

Deftnition. A connection on $E$ is a g-valued 1 -form $\theta \in A^{1}(E, \mathrm{~g})$ such that:

i) $\theta_{x} \circ \nu_{x}=\mathrm{id}$,

ii) $R_{g}^{*} \theta=A d\left(g^{-1}\right) \circ \theta$, where $R_{g}: E \rightarrow E$ denotes the action of $g$ on $E$. 
By example, in the trivial bundle $M \times G \rightarrow M$ one has the Maurer-Cartan connection defined by

$$
\theta_{(x, g)}=\left(L_{g^{-1}} \circ \pi_{2}\right)_{*},
$$

where $\pi_{2}: M \times G \longrightarrow M$ is the projection, and $L_{g^{-1}}: G \longrightarrow G$ is the traslation by the left defined by the inverse of $g$. Using this connection and an argument of partitions of unity over triviallizing open sets one can prove easily that any (differentiable) principal $G$-bundle over a paracompact manifold has a connection.

The curvature $\Omega$ of a connection $\theta$ is the g-valued 2 -form defined by

$$
\Omega\left(v_{1}, v_{2}\right)=d \theta\left(h v_{1}, h v_{2}\right),
$$

where $h$ is the horizontal component of $v . \Omega$ is invariant by the $G$-action.

(2.2) $G$ acts on the symmetric algebra of $\mathrm{g}^{*}, S\left(\mathrm{~g}^{*}\right)$ by

$$
(g P)\left(v_{1}, \cdots, v_{k}\right)=P\left(A d\left(g^{-1}\right) v_{1}, \cdots, A d\left(g^{-1}\right) v_{k}\right) .
$$

Let $I^{k}(G)$ be the $G$-invariant subset of $S^{k}\left(\mathrm{~g}^{*}\right)$. The product on $S\left(\mathrm{~g}^{*}\right)$ induces an algebra structure on $I^{*}(G)$.

Let $\theta$ be a connection on $E$ with curvature form $\Omega$, then $\Omega^{k} \in A^{2 k}\left(E, \mathrm{~g}^{\otimes k}\right)$, and as $\Omega$ is invariant and horizontal, $P\left(\Omega^{k}\right)$ is an invariant horizontal $2 k$-form so there is a $2 k$-form on $M$ which maps to $P\left(\Omega^{k}\right)$, we will denote it by the same symbol. We have the classical result (cf. [18], cap XII):

Theorem (Weil homomorphism). $P\left(\Omega^{k}\right) \in A^{2 k}(M)$ is a closed form. Let $\omega_{E}(P)$ be the corresponding de Rham cohomology class. Then:

i) $\omega_{E}(P)$ is independent of the connection, it only depends on the isomorphism class of $E$.

ii) $\omega_{E}: I^{*}(G) \longrightarrow H_{d R}^{*}(M)$ is an algebra homomorphism.

iii) if $f: N \rightarrow M$ is a differentiable map between manifolds then

$$
\omega_{f}^{*} E=f^{*} \omega_{E} .
$$

(2.3) Although $B G$ is not in general a differentiable manifold it is possible to define a Weil homomorphism

$$
I^{*}(G) \longrightarrow H^{*}(B G)
$$

For that one observes that $N G$ is a simplicial differentiable manifold and that one can extend the notions above to this more general context: a principal $G$-bundie over a simplicial manifold $M$ is a simplicial $G$-manifold $E$ and a morphism $E \longrightarrow M$ such that it is a principal $G$-bundle in each degree, $E_{n} \longrightarrow$ $M_{n}$. A connection on $E$ is a connection on $\Delta_{n} \times E_{n}$, for all $n$, compatible 
with the morphisms of $\Delta$ and $E$ (see [5], 6.2, for more details) and one defines the curvature form $\Omega$ as in (2.2). If $P \in I^{*}(G), P\left(\Omega^{k}\right)$ is a closed $2 k$-form on $M$ and defines a cohomology class

$$
\omega_{E}(P) \in H^{2 k}(\|M\|, R),
$$

verifying i)-iii) of the above theorem.

On the fiber bundle $N \bar{G} \longrightarrow N G$ we can define the following connection: Let $\theta_{0}$ be the Maurer-Cartan connection of the fiber bundle $G \longrightarrow p t$, and $q_{i}: \Delta_{n} \times N G_{n} \longrightarrow G$ the $i$-th projection of $G^{n+1}$ into $G$; we define the canonical connection by

$$
\theta=t_{0} \theta_{0}+\cdots+t_{n} \theta_{n}
$$

Now we have:

Theorem. (cf. $[5,6.13])$ There is a canonical homomorphism

$$
\omega: I^{*}(G) \longrightarrow H^{*}(B G, R)
$$

such that if $P \in I^{*}(G), \omega(P)$ is the $2 k$-form on $N G$ represented by $P\left(\Omega^{k}\right)$, where $\Omega$ is the curvature of the canonical connection. $\quad w$ satisfes:

i) $\omega(P)(E)=w_{E}(P)$, where $\omega_{E}$ is the morphism defined in (2.2) and $\omega(P)(E)$ is the characteristic class corresponding to $\omega(P)$ by (1.4).

ii) $\omega: I^{*}(G) \longrightarrow H^{*}(B G, R)$ is an algebra homomorphism.

iii) if $H \longrightarrow G$ is a morphism of Lie groups, the diagram

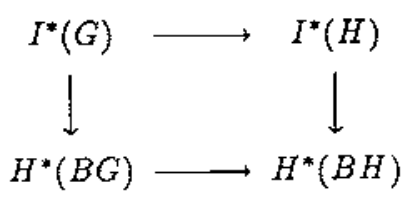

is commutative.

Remark. See [2] for another presentation of the Weil homomorphism in this general context.

(2.4) Remember that a connection $\theta$ on a principal $G$-bundle $E \longrightarrow M$ is said to be flat if its curvature form vanishes. A principal $G$-bundle admiting a flat connection will be called flat. The fiat bundles are characterized in terms of transition functions by the following result

Proposition. (cf. $[5,3.22]$ ) A principal $G$-bundle $E \rightarrow M$ is fiat if and only if there is a triviallizing open cover $U=\left\{U_{\alpha}\right\}$ of $M$ such that the transition functions $g_{\alpha \beta}: U_{\alpha} \cap U_{\beta} \longrightarrow G$ are constant.

Corollary. A principal $G$-bundle is flat if and only if admits $a G^{\delta}$-reduction.

In terms of the Weil homomorphism and using the commutative diagram $(\&)$, we deduce 
Corollary. The composition

$$
I^{*}(G) \stackrel{\omega}{\longrightarrow} H^{*}(B G, \mathbf{R}) \longrightarrow H^{*}\left(B G^{\delta}, \mathbf{R}\right)
$$

is identically zero.

(2.5) If $G$ is a compact Lie group we have the following result of Cartan

Theorem. (cf. $[5,8.1])$ Let $G$ be a compact Lie group, then the Weil homomorphism

$$
\omega: I^{*}(G) \longrightarrow H^{*}(B G, \mathbf{R})
$$

is an isomorphism.

Hence from (2.4) it follows

Corollary. Let $G$ be a compact Lie group, then the morphism

$$
H^{*}(B G, \mathbf{Q}) \longrightarrow H^{*}\left(B G^{\delta}, \mathbf{Q}\right)
$$

is zero.

(2.6) If $G$ is a complex lie group, there is a Chern-Weil homomorphism

$$
I_{\mathrm{C}}^{*}(G) \longrightarrow H^{*}(B G, \mathrm{C})
$$

similar to the Weil homomorphism. If $G$ is sernisimple with finitely many connected components then this homomorphism is bijective as onc can prove by using (2.5) applied to a maximal compact subgroup $K$ of $G$ (c.f. [22], lemma 12), so we have:

Proposition. If $G$ is a complex semisimple Lie group with finitely many connected components, then the morphism

$$
H^{*}(B G, \mathbf{Q}) \longrightarrow H^{*}\left(B G^{\delta}, \mathbf{Q}\right)
$$

is zero.

In the appendix of [22], the reader can see some other cases where $\eta^{*}$ with rationnal coefficients is zero and providing evidence of the importance of the finite coefficients in the Friedlander-Milnor conjecture. 


\section{General results}

In this paragraph we describe some general results of Milnor [22] on the isomorphism conjecture.

(3.1) First of all observe that we can reduce the study of the conjecture to the case where the coefficients are of the form $\mathbf{Z} / p \mathbf{Z}, p$ a prime number, as may be seen by using the exact sequence of homology associated to the coefficient sequence of an abelian finite group $A$

$$
0 \longrightarrow A^{\prime} \longrightarrow A \longrightarrow A / A^{\prime} \longrightarrow 0
$$

where $A^{t}$ is a nontrivial proper subgroup.

(3.2) Let $F \eta$ (or $F \eta_{G}$ if it is necessary to specify $G$ ) be the homotopy fiber of $\eta$ over $e \in G$, i.e.,

$$
F \eta=\left\{(g, f) \in G^{\delta} \times P G / f(0)=e, f(1)=g\right\}
$$

with the induced topology from the product $G^{\delta} \times P G . \quad F \eta$ is also a topological group so we can take its classifying space

$$
B \mathrm{~g}:=B F \eta
$$

The notation $B g$ is justified because $B F \eta$ only depends on the Lie algebra $g$ of $G$. In fact, if $G_{0}$ is the connected component of the identity element of $G$ and $U \longrightarrow G_{0}$ is the universal cover, the natural morphisms induce isomorphisms

$$
F \eta_{U} \stackrel{\sim}{\longrightarrow} F \eta_{G_{a}} \stackrel{\sim}{\longrightarrow} F \eta_{G}
$$

If

$$
0 \longrightarrow \mathbf{n} \longrightarrow \mathrm{g} \longrightarrow \mathrm{g} / \mathbf{n} \longrightarrow 0
$$

is an exact sequence of Lie algebras we have a fibration

$$
B \mathbf{n} \longrightarrow B \mathbf{g} \longrightarrow B(\mathbf{g} / \mathbf{n})
$$

(3.3) From the homotopy fibration

$$
F \eta \longrightarrow G^{\delta} \longrightarrow G
$$

we deduce the fibration

$$
B \mathbf{g} \longrightarrow B G^{\delta} \longrightarrow B G
$$

and so by a Serre spectral sequence argument we deduce: 
Proposition. The isomorphism conjecture is true for $G$ if and only if $B \mathrm{~g}$ has the $\mathbf{Z} / p$ - homology of a point, for every prime $p$.

As $B g$ only depends on $g$, it follows

Corollary. The isomorphism conjecture is true if it is true for the simply connected groups.

(3.4) Theorem. Let $G$ be a Lie group with solvable connected component of the identity, then the isomorphism conjecture is true for $G$.

Proof: We use induction on the dimension of $G$. By (3.3) we can assume that $G$ is simply connected, and so the first case, $\operatorname{dim} G=1$, reduces to $G=\mathbf{R}$. But we can compute explicitely the homology of $B \mathbf{R}$ and $B \mathbf{R}^{\delta}$; it suffices to remark that $B \mathbf{R}$ is contractible and that the Eilenberg-McLane homology with finite coefficients of $\mathbf{R}^{\delta}$ vanishes because it is a $\mathbf{Q}$-vector space, so the result follows in this case.

If $\operatorname{dim} G>1$, we can take a surjective morphism $G \longrightarrow \mathbf{R}$ with kernel $N$, and apply induction on the fibration

$$
B \mathbf{n} \longrightarrow B \mathbf{g} \longrightarrow B \mathbf{r}
$$

Remark. Fron the above result it follows that it suffices to prove the conjecture for the simple groups because if $\mathrm{n}$ is the solvable radical of $\mathrm{g}$ there is a fibration

$$
B \mathrm{n} \longrightarrow B \mathrm{~g} \longrightarrow B(\mathrm{~g} / \mathbf{n})=B \mathbf{s}_{1} \times \cdots \times B \mathbf{s}_{m}
$$

where $\mathbf{s}_{1}, \ldots \mathbf{s}_{m}$ are the simple algebras splitting $\mathbf{g} / \mathbf{n}$.

(3.5) In the general case, if $G$ has finitely many connected components Milnor proves:

Theorem. $([22, \S 3])$. The morphism

$$
\eta_{*}: H_{i}\left(B G^{\delta}, \mathbf{Z} / p\right) \longrightarrow H_{i}(B G, \mathbf{Z} / p)
$$

is split surjective. Similarly, $\eta^{*}$ is a split injection in cohomology.

Idea of proof: Milnor uses the Becker-Gottlieb transfer (cf. by example [19]). Let $K \subset G$ be a maximal compact subgroup of $G$, then $G / K$ is contractible (cf. [13] XV 3.I), and hence the map

$B K \longrightarrow B G$ 
is a homotopical equivalence. Let $N$ be the normalizer of a maximal torus in $K$ and consider the fibration

$$
\pi: B N \longrightarrow B K
$$

with fibre $K / N$. If $t r$ denotes the transfer morphism, then the composition

$$
H_{i}(B K) \stackrel{{ }^{r}}{\longrightarrow} H_{i}(B N) \stackrel{\pi_{*}}{\longrightarrow} H_{i}(B K)
$$

equals the product by $\chi(K / N)$, but $\chi(K / N)=1$ (cf. [14]) so we deduce that $\pi$, is a split surjection. Now it suffices to look at the commutative diagram (finite coefficients)

$$
\begin{array}{ccc}
H_{i}\left(B N^{\delta}\right) & & H_{i}\left(B G^{\delta}\right) \\
\lfloor\simeq & \downarrow^{\eta_{*}} \\
H_{i}(B N) \stackrel{\pi_{*}}{\longrightarrow} H_{i}(B K) \stackrel{\cong}{\longrightarrow} H_{i}(B G)
\end{array}
$$

and to observe that the isomorphism conjecture is true for $N$ (because there is a fibration

$$
B T \longrightarrow B N \longrightarrow B W,
$$

where $W$ is the Weil group, hence we can apply (3.4) ) to conclude the proof of the theorem.

Corollary 1. There is a direct summand of $H_{i}\left(B G^{\delta}, \mathrm{Z} / p\right)$ that $\eta_{*}$ maps isomorphically onto $H_{i}(B G, \mathbf{Z} / p)$.

Corollary 2. The homomorphism

$$
\eta^{*}: H^{i}(B G, \mathbf{Z}) \longrightarrow H^{i}\left(B G^{\delta}, \mathbf{Z}\right)
$$

is injective.

Proof: Consider the commutative diagram

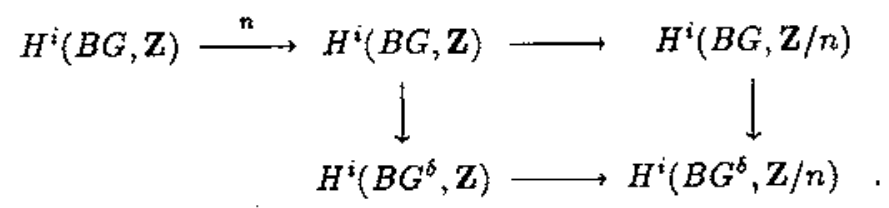

The right hand vertical arrow is injective by the theorem and the intersection of all the subgroups $n H^{i}(B G, \mathbf{Z})$ is zero, $H^{i}(B G, \mathbf{Z})$ being finitely generated, so the corollary follows. 
This result is in contrast with the effect of $\eta$ on homology. In fact, if $G$ is compact and $H_{i}(B G, \mathbf{Z})$ is free, e.g. $G=U(n)$, then

$$
\eta_{*}: H_{i}\left(B G^{\delta}, \mathbf{Z}\right) \longrightarrow H_{i}(B G, \mathbf{Z})
$$

is zero by $(2.5)$.

(3.6) The isomorphism conjecture is always true for $H_{1}(\eta)$, as can be proved easily. Some authors have studied $H_{2}(\eta)$ and $H_{3}(\eta)$ :

Theorem. Let $G$ be a simple Lie group whose Lie algebra is not one of the ten exceptionals, then $\mathrm{H}_{2}(\eta)$ is an isomorphism.

If $G$ is a complex semisimple Lie group, $H_{2}(B \mathbf{g}, \mathbf{Z})$ is isomorphic to $K_{2}(\mathbf{C})$ , [29], hence uniquely divisible [1]. In general, $H_{2}(G, Z)$ may be identified via complexification with $K_{2}(\mathbf{C})^{+}$, i.e. the conjugation stable part of $K_{2}(\mathbf{C})$, (see $[27,4.1]$ for the non-compact case and $[6,3.1]$ for the compact case), and hence the theorem follows too.

Theorem. ([24], [28]). If $G=S L(2, \mathbf{F})$, with $\mathbf{F}=\mathbf{R}, \mathbf{C}$ or $\mathbf{H}$, then $H_{3}(\eta)$ is an isomorphism.

For the real case of this theorem see [24]. The general case is treated in [28]. As in the $\mathrm{H}_{2}$ case the proof of this theorem results of the comprehension of the group $K_{3}(\mathbf{F})$. In this case Sah identifies $H_{3}(S L(2, \mathbf{F}), \mathbf{Z})$ with the group of the indescomposables $K_{3}(\mathbf{F})^{\text {ind }}$ defined by

$$
K_{3}(\mathbf{F})^{i n d}=K_{3}(\mathbf{F}) / K_{3}^{M}(\mathbf{F}),
$$

where $K_{3}^{M}(\mathbf{F})$ denotes the Milnor $K$-theory of $\mathbf{F}$.

\section{Algebraic groups}

(4.I) Let $G_{\mathbf{C}}$ be a complex algebraic group. By the comparison theorem with étale cohomology ([2]) there is an isomorphism

$$
H_{e t}^{*}\left(B G_{\mathbf{C}}, \mathbf{Z} / p\right) \cong H^{*}\left(B G(\mathbf{C})^{t o p}, \mathbf{Z} / p\right) \quad,
$$

where $B G_{\mathbf{C}}$ is the simplicial classifying group scheme of $G_{\mathbf{C}} \cdot \eta$ and this isomorphism induce a morphism

$$
H_{e t}^{*}\left(B G_{\mathbf{C}}, \mathbf{Z} / p\right) \longrightarrow H^{*}(B G(\mathbf{C}), \mathbf{Z} / p),
$$

where $G(\mathbf{C})$ is the discrete group of C-rational points of $G_{\mathbf{C}}$. In fact this map is induced by the natural map of group schemes (cf. [9])

$$
G(\mathbf{C})_{\mathbf{C}} \longrightarrow G_{\mathbf{C}}
$$

Now we can state the following conjecture: 
GENERALIZED ISOMORPHISM CONJECTURE (GIC). Let $k$ be an algebraically closed field, $n$ an integer invertible in $k$ and $G_{k}$ an algebraic group over $k$. The natural map $G(k)_{k} \longrightarrow G_{k}$ induces isomorphisms

$$
H_{e t}^{*}\left(B G_{k}, \mathbf{Z} / n\right) \stackrel{\sim}{\longrightarrow} H^{*}(B G(k), \mathbf{Z} / n) \text {. }
$$

(4.2) As in (3.2), Friedlander-Mislin prove that it suffices to consider GIC for reductive groups $([9,2.2])$ and stablish a result similar to (3.4), this time without use of a Becker-Gottlieb transfer. Using the Lang cartesian square they prove

Proposition. ([9, 2.3]). Let $p$ be a prime number and $\overline{\mathrm{F}}_{\mathrm{p}}$ the algebraic closure of the finite field with $p$ elements. If $G_{\overline{F_{p}}}$ is a connected algebraic group over $\overline{\mathrm{F}}_{p}$ then $G I C$ is true for $G_{\overline{\mathrm{F}}_{p}}$.

This result is an intermiediate step in the proof of the analogous result of (3.5):

Theorem. $\quad([9,2.5])$. Let $G_{k}$ be a connected algebraic group over $k$ and $N_{k} \subset G_{k}$ the normalizer of a maximal torus of $G_{k}$. Then the generalized isomorphism conjecture is true for $N_{k}$ and the composition

$$
H_{e t}^{*}\left(B G_{k}, \mathbf{Z} / n\right) \longrightarrow H_{e t}^{*}\left(B N_{k}, \mathbf{Z} / n\right) \stackrel{\sim}{\longrightarrow} H^{*}(B N(k), \mathbf{Z} / n)
$$

is an injection whose image is the group of stable elements $H^{*}(B N(k), \mathrm{Z} / n)^{S}$ by the $N(k)$-action.

Using the base change theorem for étale cohomology Friedlander-Mislin reduce the verification of GIC for $N_{k}$ to the cases $k=\overline{\mathbf{F}}_{p}$ or $\overline{\mathbf{Q}}$. The first of the cases follows by the proposition above. For $k=\mathrm{C}$ it is easy to prove the result so to study the case $k=\overline{\mathbf{Q}}$ they proceed in the following way: let $R \subset \mathrm{C}$ be the strict henselianization at $p$ of $\mathbf{Z}_{(p)}=\left\{\frac{m}{n}, p \mid n\right\} . R$ has residue field $\overline{\mathbf{F}}_{p}$ and $\bar{Q}$ as a field of fractions. There is a commutative diagram (cohomology with $\mathrm{Z} / n$-coefficients):

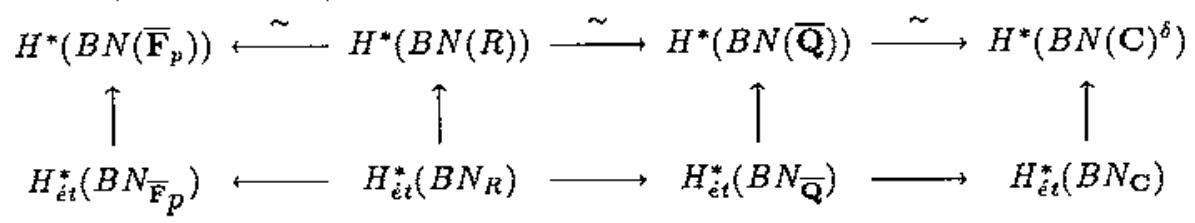

whose horizontal morphisms are isomorphisms (by Hensel lemma in the top arrows and base change in the bottom), so the result for $\bar{Q}$ follows from the result for $\overline{\mathbf{F}}_{p}$ and $\mathbf{C}$.

The identification of $H_{e t t}^{*}\left(B G_{k}, \mathrm{Z} / n\right)$ with $H^{*}(B N(k), \mathrm{Z} / n)^{S}$ is similar, though now it is not necessary to know first the case $C$ (see loc. cit. for the details).

(4.3) To finish this paragraph we remark that Friedlander-Mislin give the following characterization of GIC: 
Theorem. $\quad([9,3.2])$. The generalized isomorphic conjecture is true for $G_{k}$ if and only if for any prime $p$ and any $x \in H^{*}(B G(k), \mathrm{Z} / p)$ not zero, there is a finite subgroup $\pi \subset G(k)$ sucht that the restriction of $x$ to $H^{*}(B \pi, \mathbf{Z} / p)$ is different from zero.

As a consequence they obtain easily:

Corollary. Let $k=\cup k_{\alpha}, k_{\alpha}$ being algebraically closed fields. The generalized isomorphism conjecture is true for $G_{k}$ if and only if it is true for each $G_{k_{\alpha}}$.

This last result reduces the study of GIC to algebraic groups over a "sufficiently big" algebraically closed field for each characteristic.

\section{Stable results}

In many of the applications where one wants to know the Eilenberg-McLane cohomology of a Lie group $G$ made discrete this group $G$ is one in the classical series, $G L(n, F), U(n, F)$, etc. In such cases the study of $H_{*}\left(B G^{\delta}, \mathrm{Z} / p\right)$ may be approached in a way inspired by algebraic $K$-theory: first of all one studies stability results for the cohomology of the series and then stablishes the conjecture for the stable groups. Suslin pointed out this strategy while working on the Lichtenbaum-Quillen conjecture.

In this paragraph we relate some of Suslin's results for $G L(n, \mathbf{C})$ and similar results for other series. The field $k$ will be $\mathbf{R}$ or $\mathbf{C}$ if not specified.

(5.1) An essential result in Suslin's work is the following theorem, proved by him in a special case [35] and generalised by Gabber (unpublished) and Gillet-Thomason [10],

Rigidity theorem. Let $X$ be a smooth variety over a field $k$ and $x \in X$ a $k$-rational point. Let $\mathcal{O}_{x}^{h}$ be the henselianization of the local ring $\mathcal{O}_{x}$. If $m$ is a natural number relatively prime with the characteristic of $k$, then the natural morphism

$$
K_{*}\left(\mathcal{O}_{x}^{h}, \mathbf{Z} / m\right) \longrightarrow K_{*}(k, \mathbf{Z} / m)
$$

is an isomorphism.

Another fundamental result in Suslin's work is his stability theorem:

Stability theorem. (cf. [34]).

i) The morphisms

$$
H_{i}\left(S L_{n}(k), \mathbf{Z}\right) \longrightarrow H_{i}(S L(k), \mathbf{Z})
$$

are isomorphisms for $i \leq(n-1) / 2$. 
ii) If $k$ is an infinite field, the morphisms

$$
H_{\mathrm{i}}\left(G L_{n}(k), \mathbf{Z}\right) \longrightarrow H_{i}(G L(k), \mathbf{Z})
$$

are isomorphisms for $0 \leq i \leq n$. Moreover, the morphisms

$$
H_{n}\left(G L_{n}(k), \mathbf{Z}\right) \longrightarrow H_{n}\left(G L_{n+1}(k), \mathbf{Z}\right) \longrightarrow \ldots \longrightarrow H_{n}(G L(k), \mathbf{Z})
$$

are isomorphisms and the homology product

$$
k^{*} \otimes \ldots \otimes k^{*}=H_{1}\left(G L_{k}(k), \mathbf{Z}\right)^{\otimes n} \longrightarrow H_{n}\left(G L_{n}(F), \mathbf{Z}\right)
$$

induces an isomorphisms

$$
K_{n}^{M}(k) \stackrel{\sim}{\longrightarrow} H_{n}(G L(k)) / H_{n-1}\left(G L_{n}(k)\right),
$$

where $K_{*}^{M}$ denotes Milnor $K$-theory.

In the following paragraph we will only use ii) for $k=\mathbf{R}, \mathbf{C}$ whose proof is much more elementary as Suslin remarks.

(5.2) Suslin realizes $B \mathrm{~g}$ in the following way: fix a left invariant riemannian metric on $G$ and let $G_{e}$ be the ball of radius $\varepsilon$ centered at $e \in G$. Let $B G_{\varepsilon}$ be the geometric realisation of the simplicial set whose $p$-simplexes are the $p$-uples $\left[g_{1}, \ldots, g_{p}\right]$ such that

$$
G_{\varepsilon} \bigcap g_{1} G_{\varepsilon} \bigcap \cdots \bigcap g_{1} \ldots g_{p} G_{\varepsilon} \neq \emptyset
$$

with the usual face and degeneracy operators. Then

$$
B G_{e} \longrightarrow B G^{\delta} \longrightarrow B G
$$

is a homotopy fibration (cf. $[36,4.1]$ ). With this presentation of $B \mathrm{~g}$ Suslin proves:

Theorem. ( $\{36,4.3\})$. Let $k=\mathbf{R}$ or $\mathbf{C}$. For $\varepsilon$ sufficiently small, the inclusion

$$
B G L_{n}(k)_{\varepsilon} \longrightarrow B G L_{n}(k)^{\delta} \longrightarrow B G L(k)^{\delta}
$$

induces the zero morphism in $\widetilde{H}_{*}(-, \mathrm{Z} / m)$.

Idea of proof. By the rigidity theorem

$$
H_{\mathrm{i}}\left(G L\left(\mathcal{O}_{x}^{h}, \mathrm{~m}_{x}^{h}\right), \mathrm{Z} / m\right)=0 \quad i \geq 1
$$

where $\mathbf{m}_{x}$ is the maximal ideal of $\mathcal{O}_{x}$. 
Consider the simplicial scheme $B G L_{n} / k$, and let $X_{n, i}^{h}$ be the henselianization of its $\mathrm{i}$-th component $\left(B G L_{n}\right)_{i}=\left(G L_{n}\right)^{\times i}$ in the unit section and $\mathcal{O}_{n, i}^{h}$ be the corresponding coordinate ring. For fixed $n$, the schemes $X_{n, i}^{h}$ make a simplicial scheme and the maps

$$
X_{n, i}^{h} \longrightarrow\left(G L_{n}\right)^{X i} \stackrel{\pi_{j}}{\longrightarrow} G L_{n}
$$

define matrices $a_{j} \in G L_{n}\left(\mathcal{O}_{n, i}^{h}\right)$. Let $u_{n, i}$ be the chain

$$
\left[a_{1}, \ldots, a_{i}\right] \in C_{i}\left(G L_{n}\left(\mathcal{O}_{n, i}^{h}\right), \mathbf{Z} / m\right)
$$

where $C_{*}(-, \mathbf{Z} / m)$ is the standard complex.

By induction on $i$ and $\left({ }^{*}\right)$ for $\mathcal{O}_{n, i}^{h}$, we may prove the existence of chains

$$
c_{n, i} \in C_{i+1}\left(G L\left(\mathcal{O}_{n, i}^{h}\right), \mathbf{Z} / m\right)
$$

such that

$$
d c_{n, i}=u_{n, i}-\sum_{j=0}^{i}(-1)^{j}\left(d_{j}\right)^{*}\left(c_{n, i-1}\right) .
$$

The ring of continuous functions $\mathcal{O}_{n, i}^{\text {cont }}$ of $G L_{n}(k)^{\times i}$ is henselian hence there is a canonical map $\mathcal{O}_{n, i}^{h} \longrightarrow \mathcal{O}_{n, i}^{\text {cont }}$. Let $c_{n, i}^{\text {cont }}$ be the images corresponding to $c_{n, i}$. The group $G L\left(\mathcal{O}_{n, i}^{\text {cont }}\right)$ may be identified to the group of germs of continuous maps $\left(G L_{n}(k)\right)^{\times i} \longrightarrow G L(k)$, hence, for fixed $N>0$ so that the $c_{n, i}^{\text {cont }}$ are defined in $\left(G L(k)_{\varepsilon}\right)^{\times i}, i \leq N$, and for sufficiently small $\varepsilon$, we will have morphisms

$$
C_{\mathbf{i}}\left(B G L_{n}(k)_{\epsilon}, \mathrm{Z} / m\right) \longrightarrow C_{i+1}(G L(k), \mathbf{Z} / m)
$$

that define a homotopy to zero by the construction of the $c_{n, i}$.

Corollary. The map

$$
B G L(k)^{\delta} \longrightarrow B G L(k)
$$

induces isomorphism in homology with finite coefficients.

Idea of proof. As we have the fibration

$$
B S L(k) \longrightarrow B G L(k) \longrightarrow B k^{*}
$$

and the one corresponding to $G L(k)^{\delta}$, it suffices to see that

$$
B S L(k)^{\delta} \longrightarrow B S L(k)
$$


is an $H_{*}(-, \mathbf{Z} / p)$-isomorphism and so it suffices to prove that $\tilde{H}_{*}\left(B S L(k)_{\epsilon}, \mathbf{Z} / p\right)$ $=0$.

By the Serre spectral sequence of the fibration

$$
B S L_{n}(k)_{\varepsilon} \longrightarrow B S L_{n}(k)^{\delta} \longrightarrow B S L_{n}(k)
$$

and using the fact that

$$
H_{*}\left(B S L_{n}(k)^{\delta}, \mathbf{Z} / p\right) \longrightarrow H_{*}\left(B S L_{n}(k), \mathbf{Z} / p\right)
$$

is surjective (cf. (3.5)), we can deduce that if $i_{0}$ is the least integer with

$$
H_{i_{0}}\left(B S L_{n}(k)_{e}, \mathbf{Z} / p\right) \neq 0
$$

then

$$
H_{i_{0}}\left(B S L_{n}(k)_{\varepsilon}, \mathrm{Z} / p\right) \longrightarrow H_{i_{0}}\left(B S L_{n}(k)^{\delta}, \mathrm{Z} / p\right)
$$

is injective. But if $i \leq(n-1) / 2$, we have

$$
H_{i}\left(B S L_{n}(k)^{\delta}, \mathbf{Z} / m\right)=H_{i}\left(B S L(k)^{\delta}, \mathbf{Z} / p\right)
$$

by the stability theorem, hence $i_{0}>(n-1) / 2$ by the theorem above. Now the result follows by passing to the limit.

Using now the second statment of the stability theorem we can deduce the isomorphism conjecture for the groups $G L_{n}(k)$ in degrees $\leq n$ :

Corollary. The natural map

$$
B G L_{n}(k)^{\delta} \longrightarrow B G L_{n}(k)
$$

induces isomorphisms

$$
H_{i}\left(B G L_{n}(k)^{\delta}, \mathbf{Z} / p\right) \stackrel{\sim}{\longrightarrow} H_{i}\left(B G L_{n}(k), \mathbf{Z} / p\right) \quad i \leq n
$$

Making use of an adequate version of the above techniques Suslin and Juffrjakov prove :

Theorem. $\quad([37, \S 3])$. Let $\mathbf{H}$ be the quaternion algebra; then the natural morphism

$$
B G L(\mathbf{H})^{\delta} \longrightarrow B G L(\mathbf{H})
$$

induces an isomorphism in homology with finite coefficients.

(5.3) In [16] Jardine gives another proof of the isomorphism conjecture for $B G L(\mathbf{C})$. This new proof, of an algebraic flavor, also makes essential use of the rigidity theorem, although in this case he has not used the stability results. 
We scketch Jardine's idea: Jardine considers $B G L_{n}$ as a sheaf of simplicial sets on the category of smooth $\mathrm{C}$-schemes and defines the sheaf $B G L=$ $\underset{\lim }{\longrightarrow} B G L_{n}$. The global section functor $\Gamma_{*}$ has a left adjoint, hence there is an $\overrightarrow{a d j u n c t i o n ~ m a p ~}$

$$
\varepsilon: \Gamma^{*} B G L(\mathbf{C})=\Gamma^{*} \Gamma_{*} B G L \longrightarrow B G L
$$

corresponding to $\eta$ in the previous notations, and passing to homology there is a map

$$
\varepsilon_{*}: H_{*}\left(\Gamma^{*} B G L(\mathbf{C}), \mathbf{Z} / p\right) \longrightarrow H_{*}(B G L, \mathbf{Z} / \mathbf{p})
$$

The fiber of $\varepsilon_{*}$ in a rationnal point $x$ of a smooth variety $X$ is the map

$$
H_{*}(B G L(\mathbf{C}), \mathbf{Z} / p) \longrightarrow H_{*}\left(B G L\left(\mathcal{O}_{x}^{h}\right), \mathbf{Z} / p\right)
$$

and this map is an isomorphism by the rigidity theorem, hence $\varepsilon_{*}$ and $\varepsilon^{*}$ are sheaf isomorphisms, and so we obtain group isomorphisms

$$
\varepsilon^{*}: H^{*}\left(B G L_{\mathrm{C}}, \mathbf{Z} / p\right) \longrightarrow H^{*}\left(\Gamma^{*} B G L(\mathbf{C}), \mathbf{Z} / p\right)
$$

Finally he proves that $H^{*}\left(\Gamma^{*} B G L(\mathbf{C}), \mathbf{Z} / p\right)$ is isomorphic to $H^{*}(B G L(\mathbf{C}), \mathbf{Z} / p)$, concluding the proof.

One of the objectives of Jardine's paper is to develop the methods of simplicial sheaves on a Grothendieck topos to make sense of the program just sketched. As these are general methods he can apply them to the situation described in $\S 4$, so if $k$ is an algebraically closed field and $p$ is a prime number different from the characteristic of $k$, he obtains:

Theorem. The map

$$
H_{\dot{e} t}^{*}\left(B G L_{k}, \mathbf{Z} / p\right) \longrightarrow H^{*}(B G L(k), \mathbf{Z} / p)
$$

is an isomorphism.

In fact Jardine's proof permits to assert that the GIC is true for an algebraic group $G$ over $k$ if for every rationnal point $x$ of every smooth variety $X$ over $k$ the map

$$
H_{*}(B G(k), \mathbf{Z} / p) \stackrel{\sim}{\longrightarrow} H_{*}\left(B G\left(\mathcal{O}_{x}^{h}\right), \mathbf{Z} / p\right)
$$

is an isomorphism. One has to observe here that a so general result is unknown even for $k=\overline{\mathbf{F}}_{p}$, in which case the GIC is known to be true cf. (4.2).

(5.4) Karoubi [17] has proved the stable form of the isomorphism conjecture for the groups $S p(2 n, k)$ and $S \mathcal{O}(p, q ; k)$ and $S \mathcal{O}(n, n, \mathrm{C})$, using this time Vogtmann stability results, cf. [27]. 


\section{The Lichtenbaum-Quillen conjecture}

(6.1) Let $k$ be a field. Quillen [26] associates to the topological space $B G L(k)^{\delta}$ a new space $B G L(k)^{+}$and a map $i: B G L(k)^{\delta} \longrightarrow B G L(k)^{+}$such that

i) $\pi_{1}\left(B G L(k)^{\delta}\right) \longrightarrow \pi_{1}\left(B G L(k)^{+}\right)$corresponds to the projection $G L(k) \rightarrow$ $G L(k) /[G L(k), G L(k)]$

ii) for any local coefficient system $F$ on $B G L(k)^{+}$the morphism

$$
i^{*}: H^{*}\left(B G L(k)^{\delta}, F\right) \longleftarrow H^{*}\left(B G L(k)^{+}, F\right)
$$

is an isomorphism, and then defines the $K$-theory of the field $k$ by

$$
K_{i}(k)=\pi_{i}\left(B G L(k)^{+}\right) \quad i \geq 1
$$

(6.2) If $k$ is algebraically closed, Lichtenbaum and Quillen conjectured that the groups $K_{i}(k)$ are divisible, with zero torsion for even $i$ and equal to $W(n)$ , the Tate $n-$ twist of the roots of unity of $k^{*}$, for $i=2 n-1$. The conjecture was known to have a positive answer if $k=\overline{\mathrm{F}}_{p}$ after the determination of the groups $K_{i}\left(\overline{\mathbf{F}}_{p}\right)$ by Quillen [25].

Suslin's resuits explained in $\S 5$ give a positive answer for $k=\mathrm{C}$ :

Theorem. If $k=\mathbf{R}, \mathbf{C}$ and $G L_{n}(k)$ is the corresponding Lie group, the natural map

$$
B G L(k)^{+} \longrightarrow B G L(k)
$$

induces an isomorphism for homotopy with finite coefficients.

As was the case in (5.2) it suffices to prove the theorem for $S L(k)$, but in this case the map

$$
B S L(k)^{+} \longrightarrow B S L(k)
$$

induces isomorphism in homology with finite coefficients by the resuits of $\S$ 5 and property ii) of the +-construction. Now $B S L(k)^{+}$and $B S L(k)$ being simply-connected the result follows (cf. [23]).

$B G L(\mathbf{C})$ has the homotopy type of $B U$ hence

$$
K_{i}(\mathbf{C}, \mathbf{Z} / p)=\left\{\begin{array}{rlll}
\mathbf{Z} / p & \text { if } & i & \text { odd } \\
0 & \text { if } & i & \text { even }
\end{array}\right.
$$

Using a result of Weibel, Suslin can deduce 
Theorem. Modulo uniquely divisible groups the $K$-theory of $\mathbf{R}$ and $\mathbf{C}$ is displayed in the following table $(i>0)$ :

$\begin{array}{ccccccccc}i \bmod 8 & 0 & 1 & 2 & 3 & 4 & 5 & 6 & 7 \\ K_{i}(\mathbf{R}) & 0 & \mathbf{Z} / 2 & \mathrm{Z} / 2 & \mathbf{Q} / \mathbf{Z} & 0 & 0 & 0 & \mathbf{Q} / \mathbf{Z} \\ \downarrow & 0 & \downarrow & 0 & \text { mult.2 } & 0 & 0 & 0 & \| \\ K_{i}(\mathbf{C}) & 0 & \mathbf{Q} / \mathrm{Z} & 0 & \mathbf{Q} / \mathbf{Z} & 0 & \mathbf{Q} / \mathbf{Z} & 0 & \mathbf{Q} / \mathbf{Z}\end{array}$

(6.2) Suslin also proves in [35] that these two cases of the conjecture permits to resolve it in general:

Theorem. ([35]) The groups $K_{i}(k, Z / n)$ and the $n$-torsion groups ${ }_{n} K_{i}(k)$, only depend in the characteristic of the field.

In fact, if $k_{0} \subset k$ is an extension of algebraically closed fields we may write

$$
k=\underline{\lim } A,
$$

where $A$ runs over the finitelly generated $k_{0}$-algebras in $k$. As $k_{0}$ is algebraically closed there is a morphism

$$
A \longrightarrow k_{0}
$$

of $k_{0}$-algebras splitting the map

$$
K_{i}\left(k_{0}, \mathrm{Z} / n\right) \longrightarrow K_{i}(A, \mathbf{Z} / n)
$$

and hence the map

$$
K_{i}\left(k_{0}, \mathbf{Z} / n\right) \longrightarrow K_{i}(k, \mathbf{Z} / n)
$$

is injective. The ridigity theorem permits now assure that the morphism induced by $A \hookrightarrow k$ is the same that the one induced by (cf. loc. cit.)

$$
A \longrightarrow k_{0} \hookrightarrow k
$$

hence surjectivity follows.

Remark. As an easy corollary of the techniques above one may deduce that the groups $H_{i}\left(G L_{n}(k), \mathrm{Z} / \ell\right), \quad i \leq n$, only depend on the characteristic of $k$.

Suslin proves even more: any one of the cases $\overline{\mathbf{F}}_{p}, \mathrm{C}$ implies the truth of the conjecture, because using the rigidity theorem and the homotopy universal constructions of (5.2), he stablishes:

Theorem. (cf. [36, 3.12]). Let $k$ be an algebraically closed feld of positive characteristic $p>0$ and $L$ the algebraic closure of the quotient field $L_{0}$ of the ring of Witt vectors of $k, W(k)$. If $n$ is a prime different from $p$, there is a canonical isomorphisms

$$
K_{*}(k, \mathbf{Z} / n) \cong K_{*}(L, \mathbf{Z} / n)
$$




\section{References}

1. BASS, H. - TATE, I., "The Milnor ring of a global field," Lecture Notes in Math 342, 349-446, Springer, 1973.

2. BoTT, R., On the Chern-Weil homomorphism and the continuous cohomology of Lie groups, Adv. in Math. 11 (1973), 289-303.

3. BoTT, R., "Lectures on characteristic classes and foliations," Lecture Notes in Math 279, 1-76, Springer, 1973.

4. Dold, A. - LasiofF, R., Principal quasifibrations and fibre homotopy equivalence of bundles, $I l l . J$. Math. 3 (1959), 285-305.

5. DUPONT, I., "Curvature and Characteristic classes," Lecture notes in Math. 640, Springer, 1978.

6. DuponT, J. - PARRY, W. - SAH, C.H., Homology of classical Lie groups made discrete II : $\mathrm{H}_{2}, \mathrm{H}_{3}$ and relations with scissors congruences, Journal of Alg. 113 (1988), 215-260.

7. DUPONT, J. - SAH, C.H., Homology of euclidean groups of motions made discrete and euclidean scissors congruences, Preprint. Aarhus Univ. (1989).

8. FESHBACH, M., Some general theorems on the cohomology of classifying spaces of compact Lie groups, Trans. A.M.S. 264 (1981), 49-58.

9. Friedlander, E. - Mislin, G., Cohomology of classifying spaces of complex Lie groups and related discrete groups, Comm. Math. Helv. $\mathbf{5 9}$ (1984), 347-361.

10. Gillet, H. - Thomason, R., The $K$-theory of strict hensel local rings and a theorem of Suslin, J. Pure and Appl. Algebra. 34 (1984), 241-254.

11. HaEfliger, A., "Cohomology of Lie algebras and foliations," Lecture Notes in Math. 652, 1-12, Springer, 1978.

12. HAEFLIGER, A., The homology of nilpotent Lie groups made discrete, Astérisque 113-114 (1984), 206-211.

13. Hocischild, G., "La structure des groupes de Lie," Dunod, Paris, 1968.

14. HOPF, H. - SAMELSON, H., Ein Satz über die Wirkungsräume geschlossener Lie'scher gruppen, Comm. Marh. Helv. 13 (1940), 240-251.

15. Husemolier, D., "Fiber bundles," Graduate Texts in Math. 20, New York, 1974.

16. Jardine, J., Simplicial objects in a Grothendieck topos, Contemp. Math. 55 (1985), 193-241. 
17. KAROUBI, M., Relations between algebraic $K$-theory and hermitian $K$-theory, J. Pure and Appl. Algebra 34 (1984), 259-263.

18. KobaYASHI, S. - NomizU, K., "Foundations of differential geometry," Interscience, New York, 1969.

19. Lemaire, J.M., "Le transfert dans les espaces fibrés (d'aprés J. Becker et D. Gottlieb). Sém. Bourbaki 1975/76," Lecture Notes in Math. 567, 23-37, Springer, 1977.

20. MaCDufF, D., The cohomology of some groups of diffeomorphisms, Comm. Math. Helv. 55 (1980), 97-129.

21. Milne, J., "Étale cohomology," Princeton U.P., 1980.

22. Milinor, J., On the homology of Lie groups made discrete, Comm. Math. Helv. 58 (1983), 73-85.

23. NeISENDORfER, J., "Primary homotopy theory," Memoirs AMS 232, 1980.

24. PARRY, W. - SAH, C.H., Third homology of $S L(2, \mathbf{R})$ made discrete, J. Pure and Appl. Algebra 30 (1983), 181-209.

25. Quillen, D., On the cohomology and $K$-theory of the general linear group over a finite field, Ann. of Math. 96 (1972), 552-585.

26. Quillen, D., Higher algebraic K-theory, Proc. I.C.M. Vancouver (1974), 171-176.

27. SAH, C.H., Homology of classical Lie groups made discrete I; stability theorems and Schur multipliers, Comm. Math. Helv. 61 (1986), 308-347.

28. SAH, C.H., Homology of classical Lie groups made discrete III, J. Pure and Appl. Algebra 56 (1989), 269- 313.

29. SAH, C.H. - WAGONER, J., Second homology of Lie groups made discrete, Comm. in Alg. 5 (1977), 611-642.

30. Segal, G., Classifying spaces and spectral sequences, Publ. Math. I.H.E.S. 34 (1968), 105-112.

31. Segal, G., Categories and cohomology theories, Topology 13 (1974), 293312.

32. Segal, G., Classifying spaces related to foliations, Topology 17 (1978), $367-382$.

33. Stasheff, J., Apéndice B a [3]. Construction of $B C$, (1973), 81-85.

34. SusLin, A.A., "Homology of $G L_{n}$, characteristic classes and Milnor K-theory," Lecture Notes in Math. 1046, 357-375, Springer, 1984.

35. Suslin, A.A., On the $K$-theory of algebraically closed fields, Invent. Math. 73 (1983), 241-245. 
36. Suslin, A.A., On the $K$-theory of local fields, J. Pure and Appl. Algebra 34 (1984), 301-318.

37. Suslin, A.A., Algebraic $K$-theory of fields, Proc. ICM 1986 (1987), 222244.

38. Swrtzer, R.M., "Algebraic topology," Springer Verlag, 1975.

39. Grayson, F., On the $K$-theory of fields, Algebraic $K$-theory and Algebraic Number theory. Contem. Math. 83 (1989), 31-56.

\author{
Departament de Matemàtica aplicada I ETSEIB \\ Universitat Politècnica de Catalunya \\ Diagonal, 647 - 08028 Barcelona \\ SPAIN
}

Rebut el 2 de Juny de 1989 